\title{
La seguridad jurídica y el silogismo deductivo judicial, dentro del Neoconstitucionalismo ¿Avance o retroceso?
}

\author{
Legal certainty and judicial syllogism, within the \\ Neoconstitutionalism step forward or backward?
}

\author{
Idrovo, Diego \\ Universidad Católica de Cuenca \\ Cuenca, 010101, Ecuador \\ didrovot@ucacue.edu.ec
}

\begin{abstract}
Resumen
El constitucionalismo democrático, entendido como un proceso que modificó estructuralmente el Derecho y afirmó la doctrina jurídica del neoconstitucionalismo, se afianzó desde el final de la segunda Guerra Mundial y ha tenido una influencia en nuestra cultura jurídica. Las constituciones latinoamericanas como la colombiana, ecuatoriana, venezolana y la boliviana, son herederas de este constitucionalismo democrático y de la doctrina del neoconstitucionalismo. En el presente ensayo se realiza un análisis crítico de las aseveraciones del profesor Rodolfo L. Vigo contenidas, principalmente, en su artículo Constitucionalización y Neoconstitucionalismo: riesgos y prevenciones, en el cual alerta sobre una serie de peligros potenciales derivados del constitucionalismo que para objeto de nuestro trabajo serán dos: a) La prescindencia del silogismo deductivo judicial y, b) La pérdida de la seguridad jurídica. Comenzaré examinando la posición del profesor Vigo respecto a estos dos temas para luego realizar un análisis tomando en cuenta las posiciones de otros autores que defienden la doctrina del neoconstitucionalismo. Finalmente, pretendo ofrecer una apreciación y postura crítica frente a estos problemas utilizando la Constitución ecuatoriana como objeto de análisis y explicitar el modo en cómo el constituyente ecuatoriano ha plasmado las ideas y conceptos del neoconstitucionalismo en la Constitución de Montecristi.
\end{abstract}

Palabras clave: Ponderación, interpretación, derecho constitucional, decisión judicial.

\begin{abstract}
Democratic constitutionalism, understood as a process that structurally modified the law and affirmed the legal doctrine of neoconstitutionalism, became entrenched since the end of World War II and has had an influence on our legal culture. Latin American constitutions such as the Colombian, Ecuadorian, Venezuelan and Bolivian are inheritors of this democratic constitutionalism and the doctrine of neo-constitutionalism. In the present essay a critical analysis of the statements made by Professor Rodolfo L. Vigo mainly contained in his article Constitucionalización y Neocconstitucionalismo: risks and preventions, in which he warns about a series of potential dangers derived from the constitutionalism that for the object of our work will be two: a) The lack of judicial deductive syllogism and, b) The loss of legal certainty. I will begin by examining Professor Vigo's position on these two topics and then carry out an analysis taking into account the positions of other authors who defend the doctrine of neo-constitutionalism. Finally, I intend to offer an appreciation and critical attitude towards these problems using the Ecuadorian Constitution as an object of analysis and to explain how the Ecuadorian constituent has shaped the ideas and concepts of neo-constitutionalism in the Constitution of Montecristi.
\end{abstract}

Key words: Legal weighting, law interpretation, constitutional law, due process of law.

\section{Introducción}

El profesor Vigo manifiesta que con la llegada del Estado de derecho constitucional se generó una nueva teoría la cual se ha denominado como neoconstitucionalista, que ha sido importada desde Europa al continente latinoamericano. Esta nueva teoría, implica una noción conceptual para operar el Estado de derecho constitucional y entender conceptos categóricos como Justicia, Derecho, su interpretación y argumentación. Por tanto, existe una necesidad que abordar ciertos problemas, entre ellos, el silogismo deductivo judicial y la seguridad jurídica en el ámbito del neoconstitucionalismo.

En el presente artículo se analiza la postura del profesor Rodolfo Vigo, quien, desde hace algún tiempo, viene observando críticamente la postura de quienes defienden esta teoría del neoconstitucionalismo. Los trabajos seleccionados corresponden a la temática del iuspositivismo, iusnaturalismo, la relación entre moral y Derecho y finalmente, la teoría neoconstitucionalista ${ }^{1}$. Por ello, en este

\footnotetext{
${ }^{1}$ Véase otros trabajo de Rodolfo Vigo como (Vigo, 2012a); (Vigo,
} 
artículo se realiza una revisión bibliográfica relevante con el objetivo de condensar y extraer el mayor número de postulados del citado autor respecto a los problemas de la teoría neoconstitucionalista.

En este contexto, se escogió especialmente el artículo Constitucionalización y neoconstitucionalismo: riesgos y prevenciones, ya que este contiene una serie de premisas que se encuentran identificadas en diferentes Constituciones latinoamericanas que se inscriben en el fenómeno de la constitucionalización del ordenamiento jurídico, que concibe a las Constituciones como normas jurídicas fuertemente invasivas tanto en el ámbito público como privado.

Finalmente, el aporte de este trabajo se encamina a contribuir, en cierta medida, al debate sobre los problemas del neoconstitucionalismo y su influencia en el caso ecuatoriano, ya que el constituyente ecuatoriano, plasmó ideas y conceptos del neoconstitucionalismo en la Constitución de Montecristi vigente desde 2008.

\section{La prescindencia del silogismo deductivo judicial}

Para Vigo, la prescindencia del silogismo judicial dentro del constitucionalismo es fruto de la prevalencia del método interpretativo de la ponderación, como método racional por encima del método de la subsunción, y aunque no se elimine por completo la lógica formal en las resoluciones judiciales, es cierto que existe una prevalencia marcada de una por sobre la otra.

Así mismo, el profesor Vigo sostiene que esta apuesta por el cambio argumentativo propuesto por los neoconstitucionalistas "puede llevar al olvido del silogismo y sus reglasçon lo cual se estarían deformando las bases que çontrolan formalmente al razonamiento, incluido el prudencial o el retórico"(2008) . Vemos claramente que para Vigo, la práctica argumentativa neoconstitucionalista conlleva un peligro en contra de la lógica formal, la que por siglos ha regido la forma en la cual los jueces deben aplicar el Derecho. Nos remontamos así, al sostenimiento de las bases de un "principio monárquico como fuente formal de la Constitución, que implica reducir a ésta a un simple Código formal de articulación de poderes del Estado"(García de Enterría, 1985), en el cual, el juez es un mero aplicador de las normas establecidas por el legislador en donde los derechos y principios enunciados en la Constitución requieren de una positivización técnica por medio de leyes que operativizen dichos derechos. Podemos sostener, implicitamente, que la tesis de un juez como mero aplicador de las normas establecidas por el legislador, responde al principio de la separación de poderes, en el cual "los jueces no pueden en ningún caso rechazar la aplicación de la ley"(Guastini, 2001).

Si la tesis de la argumentación lógica formal ha sido la que se ha venido manteniendo durante siglos como método legítimo mediante el juez adopta sus decisiones, ¿Cuál ha sido la razón o razones por las cuales, este método ha sido sustituido en el neoconstitucionalismo? La respuesta es

2014); (Vigo, 2012b);(Vigo, 2010) amplia y abarca una serie de problemas que serían un tema para otro ensayo. Es por ello que para dar una respuesta acerca de la prevalencia de un método argumentativo por sobre otro, tomaremos como punto de partida el cambio radical que surgió en europa a raíz de la Primera Guerra mundial y que posteriormente se acentuó con la Segunda Guerra mundial.

Estos hechos lamentables en la historia humana, trajeron consigo una serie de cambios culturales, económicos, políticos y jurídicos en toda Europa. Los efectos derivados de estos acontecimientos, repercutieron no solo en los estados europeos sino que tuvieron un alcance mundial. A través de los sistemas regionales como mundiales de protección de los derechos humanos, se estableció la pauta inicial de un proceso mediante el cual se afianzaron los derechos individuales y se empezó a dar un esbozo y protección a los derechos sociales y colectivos.

Como sostiene Serna Bermúdez, con el objetivo de superar la çoncepción formal del Estado de derecho y su sustitución por un Estado de derecho rematerializado a partir de una comprensión de la constitución como orden o sistema de valores que se proyecta sobre el derecho ordinario o infraconstitucional"(2006), es que nace la idea del neoconstitucionalismo como un nuevo sistema jurídico que introduce en el texto constitucional valores y principios, redactados de forma explícita. El mismo autor sostiene que son tres las circunstancias que producen la constitucionalización de los ordenamientos jurídicos y que conducen a una transformación en el modo de concebir ciertas instituciones del derecho, como en este caso el silogismo lógico formal aplicado por los jueces, siendo estas: La supremacía de la constitución, el carácter normativo de las constituciones contemporáneas y la forma de cómo concebimos a dichas constituciones, es decir, como un orden valorativo proyectado sobre derechos fundamentales (Serna Bermúdez, 2006).

Es por ello, que una eventual respuesta a nuestra pregunta sería la introducción explícita de principios y valores en las constituciones, que tienen el mismo valor que el resto de normas constitucionales, es decir, que tanto los valores, principios como los derechos garantizados en la Constitución (2008), tendrían el mismo valor normativo y serían invocados indistintamente al momento de su aplicación por los jueces. Esto lo vemos reflejado en la Constitución ecuatoriana que sostiene que tanto principios como derechos son inalienables, irrenunciables, indivisibles, interdependientes y de igual jerarquía.

Ahora bien, este cambio en la estructura de la constitución trae necesariamente un cambio en la interpretación que se realiza al texto constitucional, no solo de los jueces sino todos los servidores públicos. Por ello, es que la interpretación lógico formal sufre una afectación y, en determinados casos, cede y da paso a un nuevo tipo de argumentación por medio de la cual tanto principios como valores puedan ser dotados de un contenido y aplicación normativa, lo que resulta insuficiente desde la interpretación lógico formal que los excluye por cuanto las normas jurídicas, dejando 
a un lado los principios y valores, constituyen reglas de derecho, en las que existe una relación entre una condición y una consecuencia (Kelsen, 1979). Para Kelsen, el deber ser, como consecuencia de la relación antes mencionada, no tiene un contenido moral ni axiológico, sino más bien un sentido puramente lógico, lo cual nos lleva a deducir que tanto principios como valores carecerían de esta estructura lógica formal, y no podrían ser tratados como reglas de derecho. Frente a la tesis de Kelsen, encontramos al profesor Alexy, quien manifiesta que existen dos operaciones fundamentales de aplicación jurídica: la subsunción y la ponderación (2008) en donde la estructura de la última, juega un papel decisivo al momento de dotarle de legitimidad, y donde la racionalidad debe guiar en todo momento el camino por el cual el operador jurídico toma su decisión.

Para Alexy los principios son "mandatos de optimización (...) porque pueden ser cumplidos en diferentes grados, dependiendo de las posibilidades fácticas (...) y juridicas"(2008). Por tanto, se deberá tener en cuenta por sobre todo las circunstancias y hechos especiales en cada caso, lo cual implica que la decisión que tome el juez está condicionada fuertemente a los hechos y circunstancias particulares de cada caso, volviendo imprevisible que un caso análogo, sea resuelto de la misma forma. Como sostiene Borowski, hay que tener en cuenta que la literalidad corta y carente de forma de los derechos fundamentales, todo esto ligado a una interpretación ya sea literal, genética, sistemática u objetiva de los derechos, no es suficiente y aporta muy poco al momento de establecer el contenido esencial de derechos en colisión (2003).

Dado que la subsunción representa una aplicación mecánica de la norma, dentro de un sistema normativo pleno o susceptible de ser completado, el juez tiene una posición neutra, es decir, no es ni moral ni políticamente responsable por las decisiones que toma en su resolución (Prieto, 2007).

Por el contrario, el juez, en el modelo neoconsitucionalista se convierte como señala Prieto Sanchís . en una figura moral y políticamente responsable en la medida en que (...) su decisión responda a sus propios criterios de justicia o moralidad"(2007), dentro de un sistema jurídico, en el cual la ponderación se "ha convertido en un criterio metodológico básico para la aplicación jurídica (...) de los derechos fundamentales"(Pulido, 2006).

Frente las tesis antes expuestas encontramos a autores como Juan G. Amado quien sostiene que existe un "fuerte desplazamiento de la argumentación y se sus reglas básicas", produciendo que la argumentación constitucional se convierta en metafísica y tome visos fuertemente esotéricos (2010). Siguiendo la misma idea, Kelsen también advierte de este problema, y es por ello, que en su teoría pura del derecho, establece como figura preponderante lo que denomina como la norma fundamental, la cual es la base sobre la que descansan las demás reglas de derecho de un Estado, otorgándole unidad a todo el ordenamiento jurídico. Todas las reglas de derechos están diseñadas sobre el mismo esquema, esto es, la relación entre la condición y un acto coactivo que debe ser la consecuencia (1979). Por ello, Kelsen sostiene que pueden existir actos que no tengan una significación subjetiva de una norma, como por ejemplo, que un juez declare en su sentencia que considera su decisión como justa por cuanto tiene el deber de proteger a los pobres respecto a los ricos. En este caso, dicha afirmación no tendría ninguna relación con la norma fundamental del orden jurídico, que no gozaría de una significación jurídica objetiva sino meramente subjetiva.

Debemos reconocer que en la Constitución ecuatoriana no solo encontramos principios, valores y derechos, sino que además existen también reglas. Sería absurdo sostener que desde la vigencia de la nueva constitución aprobada en Montecristi el ordenamiento jurídico no reconoce como método de interpretación o aplicación a la subsunción y que la ponderación viene a constituir el único método valido para aplicar normas de derecho.

\section{Como sostiene Ramiro Ávila:}

Una norma y un sistema jurídico debe contener tres elementos para su cabal comprensión: descriptivo, que es lo único que ha sido considerado por la ciencia jurídica tradicional, prescriptivo (los principios y, entre ellos, los derechos humanos), y valorativo o axiológico (la justicia). Sin uno de estos tres elementos, el análisis constitucional del derecho sería incompleto e inconveniente (Ávila, 2012).

De igual forma, el fenómeno del neoconstitucionalismo, trajo consigo el fenómeno denominado como activismo judicial, idea "que viene ligada estrechamente al concepto de Constitución y por ende al papel que desempeñan los jueces dentro de cada Estado en donde el papel de los jueces es de tal importancia y peso que las decisiones adoptadas por el legislador democrático pierden su carácter absoluto puesto que cuando éstas no hayan respetado los principios y garantías de rango constitucional pueden ser anuladas por los tribunales constitucionales.

Como vemos, el papel del juez dentro del neoconstitucionalismo cobra una importancia radical y preponderante, en el cual la misma Constitución le faculta y le otorga varios mecanismos para controlar la validez y coherencia de las normas; permitiéndole realizar una interpretación y aplicación del Derecho ya no solo enmarcada en la técnica de la subsunción.

El problema radica en el hecho de que la ponderación puede estar condicionada a un actuar volitivo de parte del juez, es decir, la decisión estará impregnada de un juicio de valor que deberá sopesar qué principio o valor está por encima de otro, teniendo en cuenta, los hechos particulares de cada caso. Lo cual, al parecer, afectaría la certeza con la que el juez resuelva un caso análogo. Para algunos autores como Prado Maillard, el "gobierno de los jueces es más factible en América Latina, donde el modelo presidencial está presente en todos los sistemas políticos, a diferencia de Europa, donde hay modelos parlamentarios en los que 
los tribunales constitucionales tienen menos actividad en la resolución de conflictos"(2006). Lo cual responde, como sostuvimos anteriormente, a que la forma en la que está organizado el poder, responde ya no a una separación de poderes, sino más bien al modelo de la división del poder, que implica que las leyes están sujetas a control jurisdiccional de legitimidad constitucional, de modo que los jueces pueden rechazar la aplicación de la ley inconstitucional, o hasta anularla (Guastini, 2001).

\section{La pérdida de la seguridad jurídica}

Según Vigo, otro de los problemas que identifica dentro del neoconstitucionalismo, es la pérdida de seguridad jurídica por cuanto supone que el constitucionalismo persigue fuertemente la equidad o la justicia, en donde nuevamente el rol del operador de justicia se crítica por el hecho de que éste, busca la correcta solución entre los principios y valores en tensión a través de la ponderación, buscando la mejor respuesta para cada caso.

Para Vigo, este objetivo por legitimo que parezca, vulnera un principio decimonónico como el de la seguridad jurídica, el cual establece normas previas que determinan las consecuencias que pueden derivarse de tal o cual comportamiento. Para Vigo, en el constitucionalismo se termina confiando "dogmáticamente en la razón justa del juzgador"(2008).

Pareciera ser que el problema de la pérdida de la seguridad jurídica, viene aparejado con el problema anteriormente analizado. Uno puede ser consecuencia del otro. La propia Constitución ecuatoriana establece que la seguridad jurídica es un derecho que se fundamenta tanto en el respeto de la Constitución como en la existencia de normas jurídicas previas, claras, públicas y aplicadas por las autoridades competentes. Así mismo, la Constitución establece que el sistema procesal es un medio para la realización de la justicia y que los jueces administrarán dicha justicia con sujeción a la Constitución, instrumentos internacionales de derechos humanos y a la Ley.

Vemos de esta manera que la Constitución garantiza la seguridad jurídica, es más, lo asemeja y le otorga la calidad de un derecho. Sin embargo, establece claramente que lo que se persigue dentro del sistema procesal es la justicia y le impone al juez la obligación de aplicarla, de acuerdo a tres parámetros: La Constitución, los instrumentos internacionales de derechos humanos y finalmente la Ley. Ahora bien, encontramos dentro de la Constitución una serie de mecanismo y que delimitan el camino del juez al tomar su decisión. Encontramos el derecho al debido proceso, que obliga al juez a seguir, cumplir y vigilar ciertos esenciales derechos que deben ser garantizados en todo momento. Encontramos una serie de principios de aplicación de los derechos que son de carácter general y tienen que leerse en conjunto para todos y cada uno de los derechos (Ávila, 2012), de esta forma el juez encuentra mecanismos constitucionales que le permiten motivar su decisión de forma legítima ajustándose a la normativa constitucional y legal vigente. La pregunta que surge es la siguiente: ¿Qué tipo de justicia debe buscar el juez?

Es muy difícil establecer una respuesta clara sobre el tipo de justicia que podría perseguir o consagrar una Constitución, como sostiene Rousseau: "Hubiera querido nacer en un país en el cual el soberano y el pueblo no pudiesen tener más que un solo y único interés con el objeto de que todos los movimientos de la máquina no se encaminarán nunca a otra cosa que al bien común (...), hubiera querido nacer bajo un gobierno democrático sabiamente moderado"(1981).

Para John Stuart Mill, el principio o ideal que persigue un individuo y una sociedad se basa en que el "libre desarrollo de la individualidad es uno de los principios esenciales del bienestar"(2005), fundamento básico del derecho de libertad que a su vez es la única fuente infalible y permanente del progreso que debe asegurar un Estado. Es así, que para estos autores la justicia que se busca y está garantizada por una Constitución es diferente y está basada en otros principios como la libertad individual, y el bien común. Para Michael Sandel cuando hablamos de justicia, nos referimos "no solo a cómo debemos tratarnos como individuos, sino cómo debería estar diseñada la Ley y cómo debería organizarse la sociedad"(2011). Es así, que distingue tres principales tesis sobre la justicia: Maximizar el bienestar, respetar la libertad y promover la virtud.

Ahora bien, ¿Qué tipo de justicia proclama la Constitución y debe ser aplicada por el juez? Para responder esta pregunta tomaremos en cuenta lo citado por los autores mencionados.

Si tomamos en cuenta el preámbulo, la Constitución ecuatoriana establece que el pueblo ecuatoriano -sociedadrespeta la dignidad de las personas y las colectividades, así mismo, encontramos en el art.1 la declaración de que el Ecuador es un Estado constitucional de derechos y justicia. Lo cual no presenta una claridad acerca de la justicia que el Estado garantiza y protege. Sin embargo, existen ciertas declaraciones que parecieren garantizar el bien común o bienestar por encima del interés individual.

En el artículo 83 numeral 7 de la Constitución encontramos los deberes y responsabilidades los ciudadanos ecuatorianos que, en este caso, establecen el deber de promover el bien común y anteponer el interés general al interés particular, conforme al buen vivir. Ahora bien, la Constitución consagra garantías tanto individuales como colectivas que se reconocen y protegen por igual, aunque en ciertos casos tenga prevalencia el interés colectivo por el individual y viceversa. En estos casos son los legisladores, en un primer momento, y los jueces, en un segundo momento, quienes deberán realizar un . ${ }^{a}$ nálisis racional confiando en sus propias opiniones al desarrollar o aplicar una teoría sobre la manera de interpretar un estatuto"(Dworkin, 1992).

Para Ramiro Ávila los jueces gozan de amplias facultades en el ejercicio de su competencia y jurisdicción, pero siempre están descritas en la Constitución o le Ley, es así que sostiene que "las jueces y los jueces son activos por necesidad y siempre excepcionalmente"(2012). Es por ello, 
que el ejercicio argumentativo que los jueces realizan en sus resoluciones, debe estar provisto por una forma especial de considerar tanto los hechos en debate como las normas del ordenamiento jurídico, que giran en torno al problema. Para Dworkin, el operador de justicia Juez. Hércules interpreta cada uno de ellos -estatutos- de modo que su historia sea la mejor posible, teniendo todo en cuenta"(1992). Respecto al aspecto volitivo del juez, añade que sus propias convicciones sobre la justicia o la buena política están restringidas en su propio juicio interpretativo, no sólo por el texto del estatuto sino también por una variedad de consideraciones de equidad e integridad.

Por ello, la misma Constitución establece límites a la interpretación del juez, marcando un camino que debe seguir al momento de dictar sus resoluciones, evitando el problema de la brecha interpretativa (Gargarella, 1996) a la cual la identifica como una consecuencia del poder contramayoritario que los jueces- representes del poder judicial- ejercen y terminan decidiendo sobre temas que incumben a los ciudadanos o legisladores, es decir, temas en donde la voluntad popular se debe expresar.

Ahora bien, las críticas al neoconstitucionalismo respecto a la pérdida de seguridad jurídica que realiza Vigo, responden principalmente al argumento de que en ciertos casos el juez, en vez de aplicar la normativa legal vigente, podría hacer uso de una argumentación y buscar la equidad o la justicia para resolver el caso controvertido. Sin embargo, como lo sostiene Alexy, la ponderación es utilizada principalmente en los casos difíciles en donde el ordenamiento jurídico infra constitucional es insuficiente para resolver el problema y el juez debe resolver dichos casos, a través de la técnica de la ponderación y establecer el grado de afectación en cada caso (2008). Luego, este problema de casos fáciles o difíciles no es exclusivo del constitucionalismo, pues como lo advierte Dworkin . en el positivismo jurídico encontramos una teoría de los casos difíciles"(1992) donde el juez, deberá resolver el caso ya no solo en base a un silogismo judicial, en el caso de que exista una norma aplicable que resuelva el litigio, sino teniendo en cuenta los casos análogos o remitiéndose a los principios generales del Derecho, lo cual deja por fuera al silogismo y lo coloca en una posición volitiva donde la razonabilidad del juzgador pasa a ser la herramienta interpretativa al momento de resolver un caso dificil. Cuando determinado litigio no se puede subsumir claramente en una norma jurídica, establecida previamente por alguna institución, el juez- de acuerdo con esa teoría- tiene discreción para decidir el caso en uno u otro sentido.

\section{Conclusiones}

Es verdad que la Constitución establece el derecho de la seguridad jurídica, el cual debe ser respetado y garantizado por todo servidor público- lo que incluye a todos quienes forman parte de las diferentes funciones del Estado- y por los particulares en general. La propia Constitución establece mecanismos de protección de derechos, así como, garantiza mecanismo de aplicación de derechos y los enmarca a todos estos, como de obligatorio cumplimiento a sus diferentes destinatarios.

La Constitución faculta y otorga prerrogativas a los jueces para que, en los casos en donde la insuficiencia de la norma legal es explícita, puedan hacer uso de otros mecanismos y métodos de interpretación que aseguren la búsqueda de una equidad y justicia en cada caso en concreto. Es la misma Constitución que viene a estructurar un nuevo modelo estatal, que involucra cambios en el sistema económico, político, social y jurídico, lo que hace imposible, o al menos incoherente y poco práctica, la tesis de mantener dogmáticamente principios del Estado de derecho decimonónico, que actualmente son muy poco útiles en la resolución de casos trascendentales que el juez debe resolver.

El problema de la percepción de la pérdida de la seguridad jurídica como el de la prescindencia del silogismo deductivo judicial, es fruto de la falta de concepción de la Constitución como una verdadera norma jurídica, de carácter fundamental, que materializa tanto derechos, principios como valores garantizados y reconocidos por la misma; dejando de lado al formalismo . en el cual el orden constitucional no tendrá, previsiblemente, reflejos o proyecciones importantes"(Serna Bermúdez, 2006).

Si bien el neoconstitucionalismo tiene sus inicios en Europa, principalmente en Alemania, no es menos cierto que en Latinoamérica se ha implementado este sistema hace relativamente poco tiempo y en nuestro país exactamente desde la Constitución de Montecristi de 2008. Es por ello que nos encontramos en un proceso de construcción y elaboración de nuestra propia teoría dentro de un nuevo constitucionalismo. Tenemos una ventaja que es conocer los puntos fuertes así como los problemas, que este nuevo sistema trae consigo.

Es acertada la visión del profesor Vigo, quien a través de sus diferentes trabajos ha podido identificar tensiones respecto a cómo entender el Derecho, la Justicia y el papel de los jueces en la aplicación e interpretación de la norma constitucional dentro de la teoría neoconstitucionalista con especial énfasis en el contexto Latinoamericano. Los diferentes textos analizados de Vigo, ameritan una revisión obligatoria para poder entender la forma en cómo las diferentes constituciones latinoamericanas se insertan y desarrollan, conceptos y corolarios de la teoría neoconstitucionalista.

Si la eventual pérdida de la seguridad jurídica y del silogismo formal judicial pueden verse vulnerados por el activismo judicial, estamos a tiempo de corregir esos errores dotando de herramientas teóricas y metodológicas a los operadores de justicia así como a los servidores públicos para que sus decisiones gocen de una estructura racional que finalmente las dote de legitimidad constitucional y legal. 


\section{Referencias}

Alexy, R., Carbonell, M., and Lopera, G. (2008). El principio de proporcionalidad y la interpretacion constitucional. Quito: Editor Miguel Carbonell. Ed. Ministerio de Justicia y Derechos Humanos.

Amado, Juan Antonio García y Zabala Egas, J. (2010). Prologo. Neoconstitucionalismo y Argumentacion Juridica: EDILEX.

Ávila, R. (2012). Los derechos y sus garantías ensayos escritos.

Borowski, M. (2003). La estructura de los derechos fundamentales, trad. Carlos Bernal, Bogotá, Universidad Externado de Colombia, 24.

Constitucional, T. (2008). Constitución de la república del ecuador. Quito-Ecuador: Registro Oficial, 449:20-10.

Dworkin, R. (1992). El imperio de la justicia (segunda edición ed.).(c. ferrari, trans.) barcelona. España: Editorial Gedisa SA.

García de Enterría, E. (1985). La constitución como norma y el tribunal constitucional. Madrid: Civitas.

Gargarella, R. (1996). La justicia frente al gobierno: sobre el carácter contramayoritario del poder judicial. Ariel.

Guastini, Riccardo y Carbonell, M. (2001). Estudios de teoría constitucional. Universidad Nacional Autónoma de México.

Kelsen, H. (1979). Teoría pura del derecho (16ta ed.). Trad. M. Nilve). Buenos Aires, Argentina: Eudeba.(Original en alemán, 1960).

Maillard, J. L. P. (2006). Gobierno de jueces. In Neoconstitucionalismo y Estado de Derecho, pages 171-184.

Mill, J. S. (2005). Sobre la libertad. Edaf.

Prieto, L. (2007). Derechos fundamentales, neoconstitucionalismo y ponderación judicial.

Pulido, C. B. (2006). La racionalidad de la ponderación. Revista Española de Derecho Constitucional, (77):5175.

Rousseau, J. (1981). Discurso sobre el origen de la desigualdad entre los hombres, [título original: Discours sur l'origine et les fondaments de l'inégalite parmi les hommes],[traducción: José lópez y lópez],[introducción: Jean staroninski],[1 ${ }^{\mathrm{a}}$ edición: 1754$], 2^{\mathrm{a}}$ reimpresión. Editorial Aguilar: Madrid, España.

Sandel, M. J. (2011). Justicia: ¿ hacemos lo que debemos? Debate.

Serna Bermúdez, S. (2006). Neoconstitucionalismo e interpretación. dos reflexiones en torno a la interpretación constitucional. JM Azcona Pastor/P. Torres Estrada (Comp.), Neoconstitucionalismo y estado de derecho. México: Limusa.

Vigo, R. (2008). Constitucionalización y neoconstitucionalismo: riesgos y prevenciones. op. cit, page 439.

Vigo, R. (2012a). Derecho y moral en el estado de derecho constitucional (proyecciones teóricas: iuspositivismo, neoconstitucionalismo y realismo jurídico clásico). Prudentia Iuris, 74:57-78.

Vigo, R. L. (2010). Del estado de derecho legal al estado de derecho constitucional. Revista Auctoritas Prudentium, (4):5-16.

Vigo, R. L. (2012b). De la interpretación de la ley a la argumentación desde la constitución: realidad, teorías y valoración. Dikaion, 21(1):187-227.

Vigo, R. L. (2014). Iusnaturalismo y neoconstitucionalismo: coincidencias y diferencias. Palestra Editores.

Recibido: 14 de Junio de 2016

Aceptado: 12 de mayo de 2017

Idrovo, Diego: Abogado de los Tribunales de Justicia de la República del Ecuador y Licenciado en Ciencias Políticas y Sociales por la Universidad de Cuenca, Magister en Derecho con mención en Derecho Constitucional UASB, Profesor investigador de la cátedra de Derecho Constitucional. 